\title{
Cognitive Functionality in Next Generation Wireless Networks: Standardization Efforts
}

\author{
R. Venkatesha Prasad and Przemyslaw Pawelczak, Delft University of Technology \\ James A. Hoffmeyer, Western Telecom Consultants, Inc.
}

H. Steven Berger, TEM Consulting

\begin{abstract}
This article discusses recent standardization efforts related to cognitive radio focusing on the work of IEEE Standards Coordinating Committee 41 , formerly known as IEEE 1900 . Some important tasks to be performed by the CR standardization community also are presented. These tasks will expedite the introduction of CR devices to the market while promoting a fair use of scarce radio resources. Some avenues for using the currently available standards for rapid deployment of CR devices, such as ISO standards, also are discussed.
\end{abstract}

\section{INTRODUCTION}

The idea of cognitive radio (CR) as an alternative wireless communication paradigm, capable of managing and executing itself in real-time without human intervention, was first proposed by Joseph Mitola III and Gerald Q. Maguire, Jr. [1]. In the utopian world of ubiquitous computing, each device would be equipped only with $\mathrm{CR}$, thus empowering it to communicate on any available spectrum. The question, however, is where to look for the unused spectrum with the myriads of wireless devices employed around the globe and with a status quo in spectrum regulations. As demand for radio resources increases, industry and academia are searching for efficient methods to share the spectrum - the wireless domain real-estate.

Essentially, there are two models of spectrum allocation and usage:

- Owned - where spectrum is sold or allocated to service providers or agencies who have the sole ownership of the spectrum.

The views presented here are those of the authors and do not necessarily reflect the views of the IEEE SCC41 or its projects. can Foundation with the cooperation of the Shared Spectrum Company to find the extent to which the spectrum is currently used [2]. Measurements in the $30-300 \mathrm{MHz}$ band show that utilization of some of the radio channels is less than one percent, whereas the average occupancy over all the frequency bands is only 5.2 percent. Surprisingly, the maximum total spectrum occupancy for New York City during one such measurement was found to be only 13.1 percent. Even during the peak hours of usage, one could find free spaces in the spectrum of the public bands. Thus, we may conclude that dividing spectrum into blocks is inefficient and the rigid allocation policies of regulators have resulted in an artificial scarcity of radio resources [3]. For this reason, dynamic spectrum access (DSA) concepts such as spectrum pooling and opportunistic spectrum access (OSA) are gaining momentum in academia and industry. Companies like Vanu, Inc. and Shared Spectrum Company, projects like the DARPA XG, the EU E2R, the Dutch Adaptive Ad Hoc Free Band Wireless Communications (AAF) project, and research groups in numerous universities are focusing on CR.

Since many technical, managerial, and financial aspects are associated with CR concepts (including software-defined radio (SDR)), there is a need to standardize processes, terms, and so on. Further, the efforts so far by many individual groups are incoherent due to the lack of common understanding. Therefore, strong coordination is required between regulators, academia, and product developers. To some extent, this coordination currently is lacking. To foster CR development, the IEEE Standards Coordinating Committee (SCC) 41 Standards Committee on Next Generation Radio and Spectrum Management has initiated a series of standards, the IEEE 1900 series. This article attempts to give an overview of IEEE SCC41. Some of the current thoughts with respect to harmonization of 


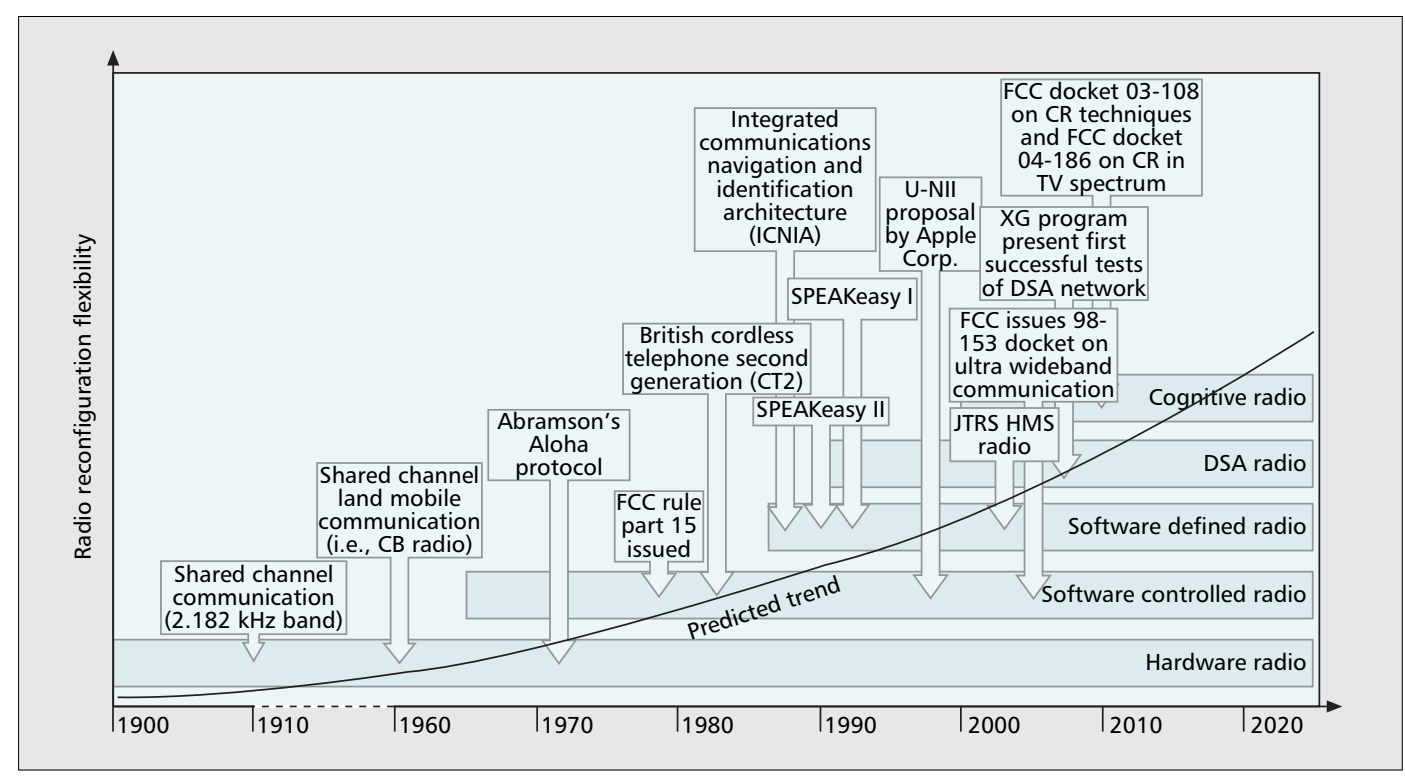

Dynamic or
opportunistic
spectrum sharing is
not a novel concept
and is probably as
old as radio
communication itself.
One of the first
communication
systems using shared
radio resources was
maritime
communication
(1910s).

Figure 1. History of CR-like systems, with a view of the future.

the IEEE 1900 series of standards and their current state of development are included here. A discussion on how to standardize CR-related processes with the currently available standardization and accreditation means is also presented here.

\section{A LOOK AT THE HISTORY OF THE DEVELOPMENT OF OPEN SPECTRUM ACCESS SYSTEMS AND REgULATIONS}

Before discussing issues related to CR standardization, it is beneficial to look briefly at the history of CR systems. Dynamic or opportunistic spectrum sharing is not a novel concept and is probably as old as radio communication itself. One of the first communication systems using shared radio resources was maritime communication (1910s). Around 1960, the Federal Communications Commission (FCC) allowed the use of shared channels, citizen band, and in land mobile communication systems. With the advent of wireless data communication, the Aloha protocol, proposed in 1970, enabled sharing of the radio channels for wireless data communication without using a centralized entity. This indirectly gave way to an FCC Rule Part 15 (1985) that described the coexistence procedures for lowpower wireless devices in the industrial, scientific, and medical (ISM) bands. Even though the Etiquette and the Listen Before Talk (LBT) protocols were not yet defined, their huge success resulted in the opening of the $5 \mathrm{GHz}$ band (UNII). The British Cordless Telephone Second Generation Standard (mid 1980s) was another example of a successful and distributed channel management technique. SDRs were developed and enabled even greater flexibility in radio access.

The first attempt at realizing SDR was in 1987 through project ICNIA by Air Force Rome Labs (AFRL), which was followed by the DARPA and AFRL SPEAKeasy I and II pro- jects (1990 and 1996). Later the U.S. Navy Digital Modular Radio (2000), and finally, the JTRS HMS Radio (2004) projects were initiated. In 2002, the FCC issued the 98-153 docket on ultrawideband (UWB) communication. Recently released FCC docket 03-122 revisited Rule 15, allowing wireless data users to share channels with radar systems on an LBT basis. Later the FCC realized that CR techniques are the future substrate that stimulates the full growth of open spectrum (see FCC dockets $03-108$ on CR technique and 04-186 on CR in TV spectrum). The previous discussion is consolidated in Fig. 1. Further information can be found in [4; 5, Chapter 1].

\section{IEEE SCC41 STANDARDIZATION EFFORTS ON COGNITIVE RADIO}

Realizing the importance of coordinated work around CR standardization, the IEEE created the IEEE SCC41 [6] to address the issues related to the deployment of next generation radio systems and advanced spectrum management. IEEE SCC41 was preceded by the IEEE 1900 task force, which initiated the IEEE standards setting effort in this area. The task force was started in the first quarter of 2005, jointly by the IEEE Communications Society and the IEEE Electromagnetic Compatibility Society. Finally on April 21, 2007, the IEEE created a new governing body for all IEEE 1900 standards and named it SCC41 on Dynamic Spectrum Access Networks.

IEEE SCC41 is divided into four working groups (WGs) and one study group, each responsible for evolving standardization processes for different aspects of CR. WGs are identified as IEEE 1900.x, where .x represents one of the WGs. Each WG shall submit a document to IEEE after finalizing the recommendations thorough discussions. After the documents are finalized, they are submitted to a vote arranged by the IEEE Standards Association (SA). During 


The framework for
interference analysis
addresses the
context of
measurements and
the purpose. Any
new adaptive system
has a trade-off
between cost and
gain. Thus, the
interference analysis
should make this
gain explicit along
with the usage
model for which
this trade-off is
accounted.

the balloting, IEEE SA members vote with their comments. Negative votes will be carefully considered by the working group, and the issues addressed by it go to the next round of discussions and/or rewriting and balloting again. Thus, a fair chance is given to interested organizations and individuals to make the standards applicable to a wide spectrum of products using CR.

\section{IEEE 1900.1: STANDARD DEFINITIONS AND CONCEPTS FOR SPECTRUM MANAGEMENT AND Advanced Radio System TeChNologies}

Many groups working on CR-related topics have defined CR and other related terms differently. Thus, IEEE decided to create a $1900.1 \mathrm{WG}$ responsible for creating a glossary of important CR-oriented terms and concepts. It further provides explanations to germinate a coherent view of the various efforts that are taking place in the broad arena of CR. The key idea is to standardize and explain technically precise definitions related to CR. In fact, $1900.1 \mathrm{WG}$ acts as a connection to the other IEEE SCC41 WGs, tying them together with common definitions of CR terms.

Some terms used in the literature have different meanings, depending on context and intent. Although the term cognitive radio has been used for years, many authors and institutions are trying to coin their own definitions and meanings. For example, Mitola III defines CR in his Ph.D. thesis as "the point in which wireless personal digital assistants (PDAs) and the related networks are sufficiently computationally intelligent about radio resources and related computer-tocomputer communications to detect user communications needs as a function of use context, and to provide radio resources and wireless services most appropriate to those needs." However, the SDR Forum explains it as "a radio that has, in some sense,

- Awareness of changes in its environment

- In response to these changes adapts its operating characteristics in some way to improve its performance or to minimize a loss in performance."

One can easily see the differences between the previous definitions. This emphasizes the importance of having common definitions and explanations, which is the role of IEEE 1900.1 WG to provide.

\section{IEEE 1900.2: ReCOMMENDEd PRACTICE fOR INTERFERENCE AND COEXISTENCE ANALYSIS}

In the light of new CR technology, many radio systems coexist, and they try to optimize the utilization of spectrum in space and time. The accurate measurement of interference has thus become a crucial requirement for the deployment of these technologies. The mandate of the 1900.2 WG is to recommend the interference analysis criteria and establish a well-thought-out framework for measuring/analyzing the interference between radio systems. New technologies, although attempting to improve spectral efficiency - by being flexible, collaborative, and adaptive - also cause disputes. Therefore, this WG intends to establish a common standard platform on which the disputing parties can present their cases and resolve them amicably.

The framework for interference analysis addresses the context of measurements and the purpose. Any new adaptive system has a tradeoff between cost and gain. Thus, the interference analysis should make this gain explicit along with the usage model for which this trade-off is accounted. Apart from the interference power measurements and the context, impact and remedies also are to be mentioned for the analysis and comparison. Finally, parameters for analysis are derived from scenarios including the context and harmful interference thresholds. Uncertainty levels in measurements are compulsorily considered in the analysis.

\section{IEEE 1900.3: ReCOMmended Practice fOR CONFORMANCE EVAluation of SOFTWARE Defined Radio Software Modules}

On the software front, IEEE 1900.3 WG is developing test methods for conformance evaluation of software for SDR devices. The aim is to define a set of recommendations that help in assuring the coexistence and compliance of the software modules of CR devices before proceeding toward validation and certification of the final devices, as laid down in the IEEE 1900.2 and 1900.A. Because SDR is an important component of future CR networks, these recommended practices should help in creating high confidence in the deployed SDR devices. These devices will have multiple layers of software, each addressing different functionalities. Therefore, it is all the more essential to test the capability of SDR devices a priori to installing the patches correctly over the air, assuring secure execution of intended functionalities.

As an illustration, consider an implementation of the SDR device specifications into a program. This can be verified with the formal verification methods. However, formal specifications for software, mostly do not exist. Therefore, testing in these cases becomes less formal by focusing only on a particular subset of device operations. The aim is to design testing procedures that will comply with the semiformal software specifications. One of the solutions is to define checkpoints (mandatory and/or obligatory) and assertions that will reflect the specifications. For these reasons, IEEE 1900.3 WG will specify device management procedures. Because many of those exist today (e.g., the Java Mobile Device Management Server APIs), 1900.3 WG will utilize other relevant standards to achieve its goal.

\section{IEEE 1900.4: COEXISTENCE SUPPORT FOR ReConfigurable, Heterogeneous AIR INTERFACES}

Many mobile devices that are used today operate on multiple wireless networks. The study of network and device architectures that can help in distributed decision making for dynamic spectrum access (DSA) is an important area - commercially, as well as academically. These procedures particularly refer to reconfigurable terminals (including SDR) capable of accessing a 
multitude of radio access technologies. The 1900.4 WG will define the overall system architecture, splitting the functionality between terminals and the network and also the information exchange between coordinating entities. Its main goal is to increase the overall system utilization of reconfigurable terminals while increasing the perceived quality of service (QoS). All 1900.4 enabled devices should operate in an opportunistic and dynamic manner such that they will not degrade the performance of primary radio access devices. The study of heterogeneity in wireless access technologies and multi-homing of the devices - with CR capability - differentiates this WG from other WGs of SCC41. At first, the 1900.4 WG will look into only the architectural and functional definitions. The corresponding protocol definitions related to the information exchange will be addressed at a later stage.

\section{IEEE 1900.A: DEPENDABILITY AND EVALUATION OF REGULATORY COMPLIANCE FOR RADIO SYSTEMS WITH DYNAMIC SPECTRUM ACCESS}

The topic of the IEEE 1900.A study group is certification of DSA devices. Devices with potential cognitive capability bring new challenges for the certification process. To prove that a radio device always will remain within operational boundaries is more difficult compared to traditional radios. Future hardware vendors must know the design methodologies and testing procedures to affirm that their devices will not interfere with a primary user of a given frequency channel. Many technical studies are involved in this study group such as hazard analysis, listing potential causes for out-of-compliance transmission and description of previous behavior-based certification efforts. In fact, its most important task is to standardize the dependability of a radio system vis-à-vis quantifying the level of trust one has.

Different levels of trust can be defined for a particular spectrum based on its primary user. As an example, if a CR radio uses frequencies assigned for avionics, it must have a high level of confidence in its capabilities to detect the activities of the primary users. The capability assessment of a radio system to adhere to the regulations is the main focus of 1900.A.

The advantage of having this study is manifold. For example, regulators may use these recommendations before certification, whereas manufacturers may use them for the production of systems that conform to the regulations. IEEE 1900.A has currently drafted a project authorization request (PAR) for submission to the IEEE Standards Association to become another independent WG within IEEE SCC41 project.

\section{RELATEd Standardization EFFORTS}

Other IEEE projects related to next-generation radio, like IEEE $802 .\{18,19,20,22\}$ are the sources of expertise for IEEE SCC41. As mentioned earlier, because active dialogue between different standard bodies is crucial at this stage of CR development, IEEE SCC41 has initiated cooperation with the FCC, Federal Office of
Communication [UK] (OFCOM), SDR Forum, and OMG Forum, to name a few. The relation between different WGs of IEEE SCC41, as well as different standardization bodies within and outside IEEE are depicted in Fig. 2.

\section{ADVANCES IN IEEE 1900.1: A BRIEF DisCussion of Cognitive RADIO FUNCTIONALITY AND SOME Definitions}

As mentioned earlier, there are many views on what a CR is, and at times they conflict. Probably, the simplistic meaning of the term is a radio that adapts to its surroundings. Many adjectives were added later, often connecting CR with artificial intelligence, machine learning, and so on. Now the horizon of CR is expanding with cognition entering many layers of the communication stack. Therefore cognitive functionality is taken as the basis here, whether the cognition is in the radio, in the higher layers, or both. The idea is to make communication devices tune their radio and higher layer functions to utilize the available spectrum efficiently. For academic purposes, the unapproved (at the time of the writing of this article) definition of CR is mentioned here from the IEEE 1900.1 WG draft: "CR is:

- Radio in which communication systems are aware of their environment, internal state, and location and can make decisions about their radio operating behavior based on that information

- Cognitive radio, as defined in the first, that utilizes software defined radio, adaptive radio, and other technologies to autonomously adjust its behavior or operations to achieve the desired objectives"

In this context, CR is not limited to only the radio layer. Until the time of having an agreedupon definition of CR from the IEEE 1900.1 draft standard, an alternative term called cognitive functionality in wireless communication network (CFWCN) is used here to represent the art of efficient use of spectrum. This cognitive functionality may be spread across the layers of the communication architecture, resulting in coordination among the layers for an efficient use of available spectrum. The implementation of cognitive functionality includes technical, policy, and regulatory issues.

Figure 3 explains the basic functional blocks of a CFWCN node. Specifically, apart from a reconfigurable radio, a CFWCN node has various other components. The sensing block and policies block (if available) are extensively used in deciding the availability of spectrum. These blocks also help to drive the learning and reasoning functions. The learning and decision blocks may be implemented with fuzzy logic or neural networks. The decision database along with the input from the sensing block and policies block drives learning. The end result is that the radio is configured based on input from different layers of the communication stack, as well as the environment. Moreover, the view of IEEE 1900.1 WG is that the term cognitive radio used in the literature generally means cognitive radio
Now the horizon of

$C R$ is expanding with

cognition entering

many layers of the

communication

stack. Therefore

cognitive

functionality is taken

as the basis here,

whether the

cognition is in the

radio, in the higher

layers, or both 


Accreditation is to
provide confidence
in CABs through its
control function.
Similarly, ISO/IEC
17024:2003
specifies require-
ments for
organizations
certifying the
personnel, who in
turn oversee
conformity tests.

Accreditation is to

provide confidence

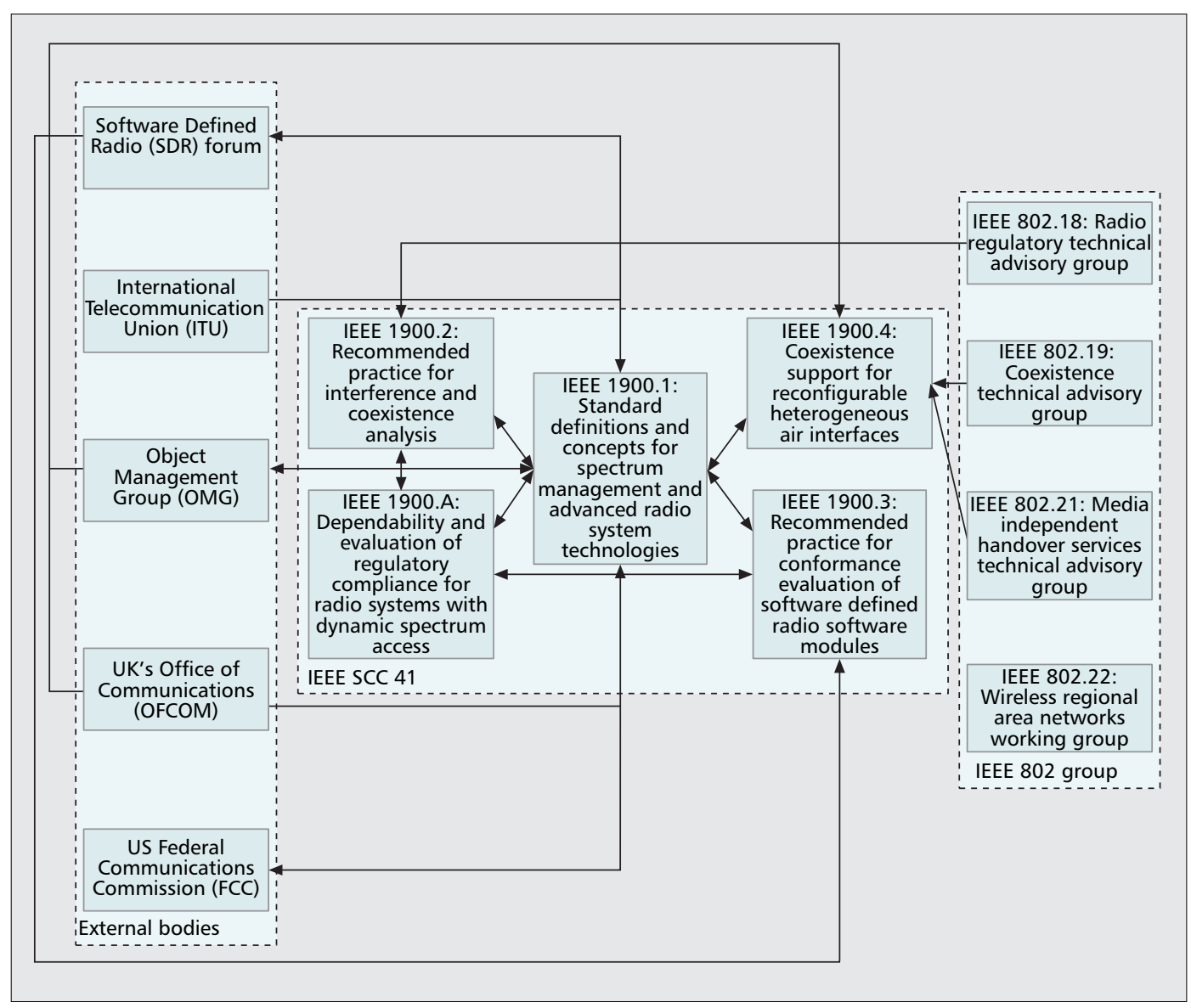

Figure 2. Nesting of various IEEE SCC41 projects and their relation with other external organizations. systems - this has been reflected in the contribution from IEEE 1900.1 to the initially drafted study questions on CR to the International Telecommunication Union-Radiocommunication (ITU-R) Study Group 8, Working Party 8A (approved later).

In fact, IEEE 1900.1 has recognized the previously mentioned view that cognition, awareness, adaptability, and so on, may lie outside the boundaries of the radio. This aspect is highlighted by the additional word system.

In the following, other important definitions from the current IEEE 1900.1 draft [7] are reproduced. We note that some of these definitions were developed after many rounds of discussion and are not yet approved by IEEE officially; the authors claim no rights whatsoever on any of these definitions.

Dynamic spectrum access: The near-real-time adjustment of spectrum resource usage in response to changing circumstances and objectives; including interference experienced or created; changes of the radio state (operational mode, battery life, location, etc.); and changes in environmental/external constraints (spectrum, propagation, operational policies, etc.).

Opportunistic spectrum access: The method by which spectrum users operating on a secondary (and possibly unlicensed) basis within a frequency band with designated primary (and possibly licensed) users exploit unused in-band segments for their own purposes without causing interference to the active interference-intolerant primary users for the duration of the availability of the spectrum in question.

Software-defined radio: A type of radio in which some or all of the physical layer functions are software defined. For example, it is feasible that a radio has two or more modulation schemes, each of which is implemented in hardware. However, software control is used to switch the radio characteristics. In the FCC and ITU-R definitions, if such control is used in a radio, it is considered to be SDR, even if the radio signal processing is done entirely in hardware. IEEE 1900.1 does not consider software control to be SDR.

Cognitive radio network: A network capable of establishing links between its cognitive radio nodes to establish connectivity and to adjust its connectivity to adapt to changes in environment, topology, operating conditions, or user requirements.

Quality of detection: A metric that implies the quality of identification of spectral opportunities that introduces a particular level of interference to the primary users of the channel but not limiting a secondary network from accessing it while it was vacated by the primary network [8].

Spectrum broker: A device responsible for dynamic assignment of particular frequency channels to secondary devices [8].

Spectrum etiquette: A set of rules, policies, and procedures that govern the behavior of a collection of wireless networks. 
Table 1 lists categories and classes of different future adaptive radio devices. This simplistic comparison tries to show the differences between them. Some confusion still subsists in the CR community on how to classify different devices/systems.

\section{CONFORMITY ASSESSMENT, ACCREDitation, AND Certification}

Because the next generation radio deals with SDR and CR devices that are capable of downloading software to reconfigure themselves, it is beneficial to have a look at the existing standardization policies for software certification, accreditation, and so on. The new standards benefit immensely by adopting relevant standardization processes from the existing ones. For background, as an example, some of the International Standard Organization (ISO) procedures are briefly revisited here.

Conformity tests are important for smooth interworking and a broader acceptance of the devices. There are two types of certification within ISO:

- Certifying the process

- Certifying the product

The ISO 9000 series standards focus on the capabilities of an organization to maintain the quality of various processes, and in turn its ability to maintain the quality. Conformity assessment bodies (CABs) are organizations that provide the testing, inspection, management system certification, personnel certification, product certification, and calibration. CABs should first conform to ISO/IEC 17011:2004, which specifies general requirements. ISO 17011 standards provide requirements for the peer evaluation for mutual recognition between accreditation bodies. Therefore, conformity tests done by a CAB may be directly admissible in any other geographic location. Thus the regulators who allow CABs to certify and calibrate devices must enforce ISO 17011 first. This avoids repeated conformity assessment by various organizations in different countries.

Accreditation is to provide confidence in CABs through its control function. Similarly, ISO/IEC 17024:2003 specifies requirements for

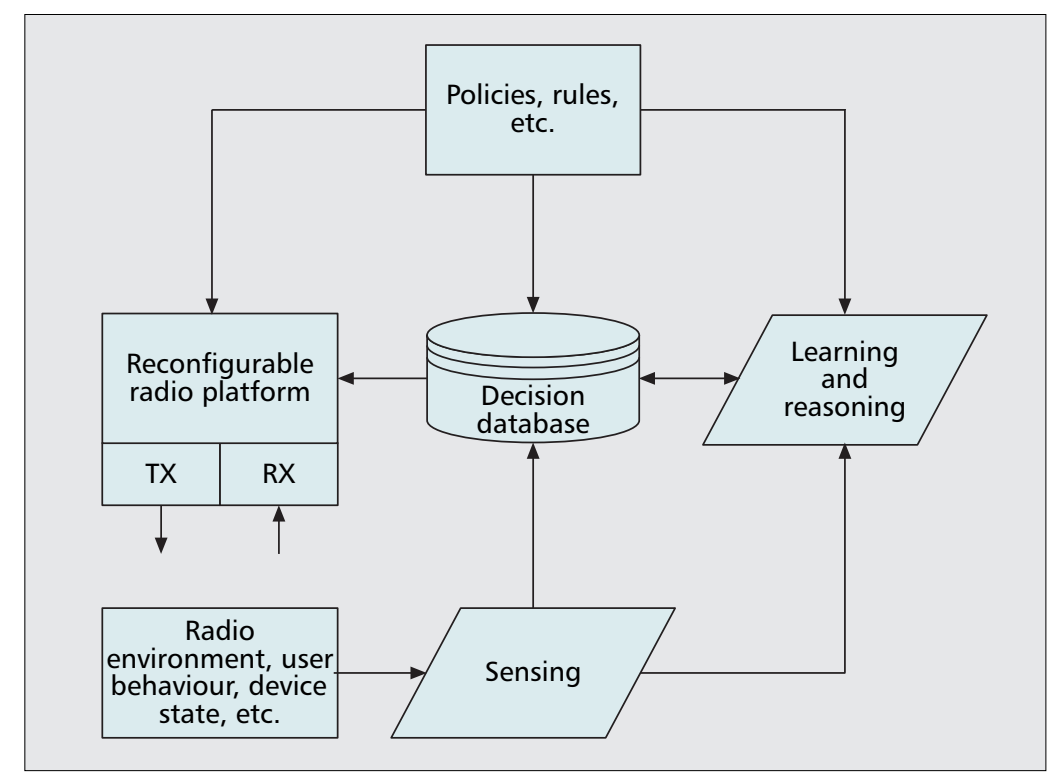

Figure 3. Cognitive radio functionality.

organizations certifying the personnel, who in turn oversee conformity tests. ISO/IEC 17025:2005 specifies the general competence requirements for the personnel to carry out these tests and calibrations. It is desired that CR devices should first get the approval from the CABs and from individuals who have been certified by ISO. One important standard to mention in this context is ISO/IEC 9126-(1-4):2001-2004, which is an international standard for the evaluation of software. This standard is divided into four parts:

- Quality model

- External metrics

- Internal metrics

- Quality in use metrics

Thus, the standard captures all the necessary tools to evaluate the software used in critical settings. The personnel have the access to the regulations on interference and requirements from spectrum regulators regarding the use of various spectrum to quantify the metrics mentioned previously.

The certification that the devices are tamperproof is crucial for wide use of CR devices. This

\begin{tabular}{|c|c|c|c|}
\hline Type of radio & Platform & Reconfiguration and adaptability & Intelligence \\
\hline Hardware & Hardware & Minimal & None \\
\hline Software & Hardware/software & Automatic & Minimal \\
\hline Adaptive & Hardware/software & Automatic/predefined & Minimal/none \\
\hline Reconfigurable & Hardware/software & Manual/predefined & Minimal/none \\
\hline Policy-based & Hardware/software & Manual (database)/automatic & Minimal/none \\
\hline Cognitive & Hardware/software & Full & Artificial/machine learning \\
\hline Intelligent & Hardware/software & Full & $\begin{array}{l}\text { Machine learning/use of } \\
\text { prediction for decision }\end{array}$ \\
\hline
\end{tabular}

Table 1. Comparison of different types of adaptive radio devices. 
The certification that

the devices are

tamperproof is

crucial for wide use

of $C R$ devices. This is

mandated by the

fact that $C R$ devices

access shared

resources. An

example for this is

the USA Trusted

Computer System

Evaluation Criteria. is mandated by the fact that CR devices access shared resources. An example for this is the USA Trusted Computer System Evaluation Criteria [10]. There already is some discussion on this topic using Software Considerations in Airborne Systems and Equipment Certification standard DO-178B of the Radio Technical Commission for Aeronautics. Five levels of software failure risks are identified there. Depending on the risk levels, design and validation processes are recommended. It is believed that these standards, which are in force, may expedite the process of standardization of CR devices.

\section{CONCLUSIONS}

In this article, the efforts of IEEE SCC41 were introduced. An account of the operation of various IEEE SCC41 working groups that are focused on CR was given. The operational domain of each of the projects was described in detail. CR functionality and definitions of some terms relevant to CR were presented. As a starting point, the applicability of existing ISO to the CR standardization process with accreditation procedures and certification were introduced. It is believed that this article will be helpful for researchers, standards bodies, and organizations working on cognitive radio.

\section{ACKNOWLEDGMENTS}

Part of this research was performed in the Adaptive Ad Hoc Free Band Wireless Communications (AAF) and Magnet Beyond and Personal Network Pilot (PNP 2008) projects, funded respectively by the Freeband program of the Dutch Ministry of Economic Affairs and the EU. The authors thank Richard Roy, A. Slingeland from TU Delft, and S. Vijay of Esqube, Bangalore, India for fruitful discussions while writing this article. The authors are indebted to C. Jackson for permitting us to utilize some parts of his article and anonymous reviewers who helped correct this article.

\section{REFERENCES}

[1] J. Mitola III and G. Q. Maguire Jr., "Cognitive Radio: Making Software Radios More Personal," IEEE Pers. Commun., vol. 6, no. 4, Aug. 1999, pp. 13-18.

[2] M. Vilimpoc and M. McHenry, 2006, Dupont Circle Spectrum Utilization During Peak Hours; http://www. newamerica.net/files/archive/Doc File 183 1.pdf

[3] C. Ting, S. S. Wildman, and J. M. Bauer, "Government Policy and the Comparative Merits of Alternative Governance Regimes for Wireless Services," Proc. IEEE DySPAN '05, Baltimore, MD, Nov. 8-11, 2005, pp. 401-19.

[4] C. Jackson, "Dynamic Sharing of Radio Spectrum: A Brief History," Proc. IEEE DySPAN '05, Baltimore, MD, Nov. 8-11, 2005, pp. 445-66.

[5] B. A. Fette, Ed., Cognitive Radio Technology, Elsevier, 2006.

[6] IEEE 1900 Std. Committee,IEEE SCC41 home page: http://www.scc41.org

[7] IEEE, "Standard Definitions and Concepts for Spectrum Management and Advanced Radio Technologies," P1900.1 draft std., v. 0.31, June 2007.

[8] P. Pawelczak, G. J. Janssen, and R. V. Prasad, "Performance measures of Dynamic Spectrum Access Networks," Proc. IEEE GLOBECOM '06 San Francisco, CA, Nov. 27-Dec. 1 2006; http://dutetvg.et.tudelft. $\mathrm{nl} / \sim$ przemyslawp/files/ prfrmnc.pdf
[9] M. M. Buddhikot et al., "DIMSUMNet: New Directions in Wireless Networking Using Coordinated Dynamic Spectrum Access," Proc. IEEE WoWMoM '05, Taormina -Giardini Naxos, Italy, June 13-16, 2005.

[10] DoD 5200.28-STD Trusted Computer System Evaluation Criteria, May 1985; http://csrc.nist.gov/publications/history/dod85.pdf

\section{BIOGRAPHIES}

R. VENKATESHA PRASAD (vprasad@ewi.tudelft.nl) obtained his Bachelor's degree in electronics and communication engineering (ECE) from the University of Mysore, India, in 1991. He received his $M$. Tech. degree in industrial electronics in 1994 and his Ph.D. degree in 2003, from the University of Mysore and the Indian Institute of Science (IISc) Bangalore, respectively. Since 2005 he has been with the Wireless and Mobile Communications Group at Delft University of Technology, working on the EU funded projects MAGNET/MAGNET Beyond and PNP 2008, and guiding students. He is also a consultant to Esqube Communication Solutions Pvt. Ltd. on a part-time basis. During 1994 and 1996 he worked as a consultant and project associate for ERNET Lab of ECE at IISc. While pursuing his Ph.D. degree from 1999 to 2003, he also worked as a consultant for CEDT, IISC, Bangalore in VolP application development, as part of a Nortel Networks sponsored project. From 2003 to 2005 he headed a team of engineers at Esqube for the development of various real-time networking applications.

PRZEMYSLAW PAWELCZAK (p.pawelczak@ewi.tudelft.nl) gradu ated (cum laude) in electronics and telecommunications engineering from Wroclaw University of Technology, Poland, in 2004. Since 2005, he has been pursuing his Ph.D. degree at Delft University of Technology in the field of dynamic spectrum access networks. During the fall of 2007, he was a visiting scholar at the Connectivity Laboratory of the University of California, Berkeley. Between 2004 and 2005 he was a staff member at Siemens Software Development Center, Wroclaw, Poland. He is the originator and organizing committee member of IEEE CogNet workshops, collocated with IEEE ICC 2007 and 2008. He is a member of the IEEE Technical Sub-Committee on Cognitive Networks and the IEEE SCC41 Standardization Committee.

JAMES A. HOFFMEYER (jhoffmeyer@ieee.org) is president of Western Telecom Consultants, Inc. His current areas of interest include cellular systems, software-defined radio, cognitive radio, radio regulation, and international telecommunications standards development. He was previously employed by the National Telecommunications and Information Administration, where he managed a group of up to 25 engineers and scientists. He has over 22 years experience in wireless telecommunication. He has held numerous standards leadership positions as chair of the predecessor of the SDR Forum and as chair of working groups in the International Telecommunication Union (ITU) and in the IEEE Standards Association (SA). He chaired the working group that developed the ITU-R vision for the future development of next-generation commercial wireless telecommunication systems. He is a member of the IEEE ComSoc Standards Board. He has published over 40 technical papers and reports, and has developed more than 45 input contributions to the ITU and IEEE SA units.

H. Steven Berger (stephen.berger@ieee.org) is president of TEM Consulting, an engineering services and consulting firm dealing in regulatory compliance, wireless, and EMC. $\mathrm{He}$ is the current co-chair of IEEE SCC41, Dynamic Spectrum Access Networks, and chair of the IEEE EMC Society Standards Development Committee. He is also President of the International Association of Radio and Telecommunications Engineers. Before forming TEM Consulting, he was a project manager at Siemens Information and Communication Mobile in Austin, Texas, where he was responsible for standards and regulatory management. He has worked in EMC and RF safety for 20 years. More recently, he has been active in the area of disability access to telecommunications and information technology products. He has written numerous technical papers and holds several patents in the area of wireless and EMC. 